\title{
Ontology based user modeling for personalization of grid learning services
}

\author{
Guy Gouardères ${ }^{1}$, Emilie Conté ${ }^{2}$, Saber Mansour $^{3}$, Liana Razmerita ${ }^{4}$, \\ Laboratoire LIUPPA - Université de Pau et des Pays de l'Adour, France \\ 1 guy.gouarderes@iutbayonne.univ-pau.fr
}

2 emilie.conte@iutbayonne.univ-pau.fr

3 saber.mansour@iutbayonne.univ-pau.fr

INRIA, Projet ACACIA, Sophia-Antipolis, France

4 liana.razmerita@inria.fr

\begin{abstract}
The paper shows how semantic grid learning services will support a user-centered, personalized, contextualized and experiential based approach for ubiquitous learning. In order to allow personalized learning processes, we need to study and define methodologies for representing, through adequate knowledge structures, such as ontologies, both the area (the learning context) and the learner. In this paper we will focus on the role of personalized ontologies for a new generation of intelligent services, and more specifically, about their role for Grid Learning services in ELeGI.
\end{abstract}

\section{INTRODUCTION}

One of the long term objectives of Elegi FP6 project is to study how the Semantic Grid might enable to build a seamless intelligence for learning services on the Grid [13]. We believe that the seamless intelligence of learning services will pool user-centered concepts such as: Peer-to-Peer communication ${ }^{1}$, affective computing, serendipity with other socio-centered concepts such as: collective intelligence, ubiquitous computing and amorphous computing.

The "Learning Grid » paradigm aims at making use of the collective intelligence and the personalized use of a range of available and potential Grid Learning services. That implies that these services are significantly centred on exchange, negotiation, dialogue within and among virtual, evolutionary and pervasive communities for whom underlying universal driving mechanism learning is is viewed as a paradigm shift [1]. This ethno-centred vision can not exist without a techno-centred evolution of services, semantics and standards. The way to meet this strategic and innovative breakdown follows the multiple paths of a global challenge of which some parts are adressed in the paper:

collaboration as a basis for e-learning,

prominent role of ontologies ,

omnipresence of agents as an ubiquitous layer for dynamic service generation and dissemination, cognitive environments as a structure to represent the partners in conversations,

customization of the services for individual students,

use of Peer-to-Peer communication way.

The paper is thus structured in 4 sections. The first section presents the rationale of e-Qualification as a transverse Grid Learning Service (GLS) in ELeGI. In the second one we describe the pragmatics for an ontology based user modeling. The third section presents the use of ontologies to apply the e-Qualification in the use case ASIMIL ${ }^{2}$. We end by drawing some conclusions and planning future work.

\section{1 e-Qualification as a High Level Grid Learning Service}

e-Qualification is designed as a cognitive prosthese to be plugged on various learning services to qualify, manage and keep members in their convenient virtual community ${ }^{3}$. By virtue of a largely reusable explicit formal model of shared e-Portfolio, team members attempt to manage general responsibilities and commitments to each other in a coherent fashion that facilitates recovery when unanticipated problems arise. Moreover, we have chosen to take advantage of the Grid architecture and to work in a Peer-to-Peer (P2P) way. Therefore, agent frameworks used

\footnotetext{
${ }^{1}$ Peer-to-Peer is a communication model in which each party has the same capabilities and either party can initiate a communication session. Is opposed to the Client/Server model

2 ASIMIL: Aero user friendly SIMulation based dIstant Learning, N ${ }^{\circ}$ IST-1999-11286. FP5 http://www.cordis.lu/ist/projects.htmhttp://www.asimil.com (see §2.2)

${ }^{3}$ in the EleGI context, e-learning is become a clearly social activity which occurs in dynamic virtual communities
} 
for e-Qualification have to incorporate explicit general models of teamwork, mobility, and resource control appropriate for learning evaluation scenarii.

To apply the e-Qualification, it is necessary to belong to an adapted context which is described in the following paragraph.

\section{2 e-Qualification objectives}

To start a learning process, the learner must belong to at least a community. Each trainee is represented by an agent in his community and has an e-Portfolio (kind of dynamical curriculum vitae). Some rules have to be defined such as the criteria which allow a trainee to integrate a community. Everything will be based on the e-Portfolio. It is important to precise that all the members of a community have a part of their e-Portfolio similar to the other ones. This common part will be called the "signature".

The trainee will learn during formal learning process (simulation, $M C Q$, etc...) and with communication with the orther learners, tutors during debriefings (informal learning process). The e-Qualification has two functions :

help the trainee move inside his community : during debriefing, the «peer » associated to the user will learn from the other « peer » and then will transmit this new knowledge to the user.

Find the best community for the user (in fact, the best group of abilities among the training area) : by using a matchmaking process with the signature of the peer (trainee) who is searching a new community, and some other peers. When there is an overlay of two signatures, it means that the peer (who is searching) can integrate the community of the other one.

According to the definition of $\mathrm{VLC}^{4}$ in EleGI, we introduce Virtual Learning Group Communities (VLGC) as teams of cognitive agents working together with « users in the loop » to achieve their goals (i.e. e-Qualification of what they have learned together).

Stating that the collaborative learning is a teaching

strategy in which "both teachers and learners are active participants in the learning process", we will plan to improve this process for the informal learning situations.

In $\mathrm{VET}^{5}$, collaborative learning occurs in the phase of informal learning called Team Debriefing in ASIMIL [ 2] (group \& individual evaluation in team training). Learners share ideas as know-how, form the previous sessions on the trainer, and criticize each other's performances. In this type of environment the students become a "community of learners". They begin to view their own thoughts and mastering as meaningful to the whole community.

This community of learners is ranked into group of abilities: This is what we have called a VLGC.

To conclude, a VLC dedicated to VET or training is composed of various VLGC for monitoring Groups \& individual knowledge acquisition in P2P mode. This mode of group of trainees monitoring has been added because it was not currently planned in VLC. Moreover, all the GLS serving for both formal and informal learning are embedded into the Gride-Card (ubiquituous generic interface VLGC) [3]. The Grid-e-Card provides a pervasive basic services of sensors and effectors to support broadcast communications among VLGC in P2P mode [4].

\section{3 e-Portfolio}

The key concept of a virtual community usually involves some notion of shared knowledge, goals, and intentions that function as the glue that binds team members together. This will be done by a new design for the former portfolio or Electronic Portfolio. For e-Qualification, the e-Portfolio is an interactive process of documenting and evaluating achievements and improvements in student learning. This new version of e-Portfolio is based on two paradigms: The user model ontology and the team of P2P mobile agents we should make the most with the changes of the shared knowledge using this model of ontology [5]. The user model ontology facilitates a) sharable specification of the functionalities of the virtual learner agent; b) fluent communication between the user and other agents. The example of a user model ontology and user pedagogical agent shows that with the help of ontology, standards for sharable and reusable agents in learning support systems may be established [11].

\subsection{Learning Grid scenario: e-Qualification services in ASIMIL}

In the following part, we present a scenario of use of an application from aerospace domain called ASIMIL (A Network Distributed Simulator Training System). Grid computing has as an ultimate objective to increase business efficiency and it is an adapted infrastructure for advanced applications in aeronautics and other application domains. ASIMIL has to be adapted for the use in the Semantic Grid environment. However the question on how to realize the vision of Semantic Grid for this application is still an open question. We advance the idea of using ontologies for ASIMIL application scenario, it is a first step for making it fit for the Semantic Grid vision. The use of ontologies for modeling the user and the application domain would facilitate the communication of the various agents that are populating ASIMIL. Many learning processes supported by ASIMIL relate to a dynamic learner profile and associated user modeling processes.

\section{ONTOLOGY BASED USER MODELING}

The first ideas of using ontologies for learner modeling have been reported by Chen \& Mizoguchi (1999). Kay (2001) also argues for the use of ontologies for reusable and "scrutable" student models. More recently the idea of using sharable data structures containing user's features and preferences was proposed in order to enable

\footnotetext{
${ }^{4}$ VLC : Virtual Learning Community

${ }^{5}$ VET : Vocational and Educational Training
} 
personalized interactions with different devices for the benefit of the users. For this purpose, a user modeling markup language for ubiquitous computing built on XML technology has been proposed as a platform for communication by Heckmann \& Krueger (2003). The definition of the user ontology captures rich metadata about the employee's profiles comprising different characteristics such as: name, email, address, competencies, interests, qualifications, preferences, but also a behavioral profile specific to the user user interaction among competence groups in VLGC. A partial view of the user ontology using a graph based representation using Kaon ${ }^{6}$, Ol-Modeler is represented in Figure 1.

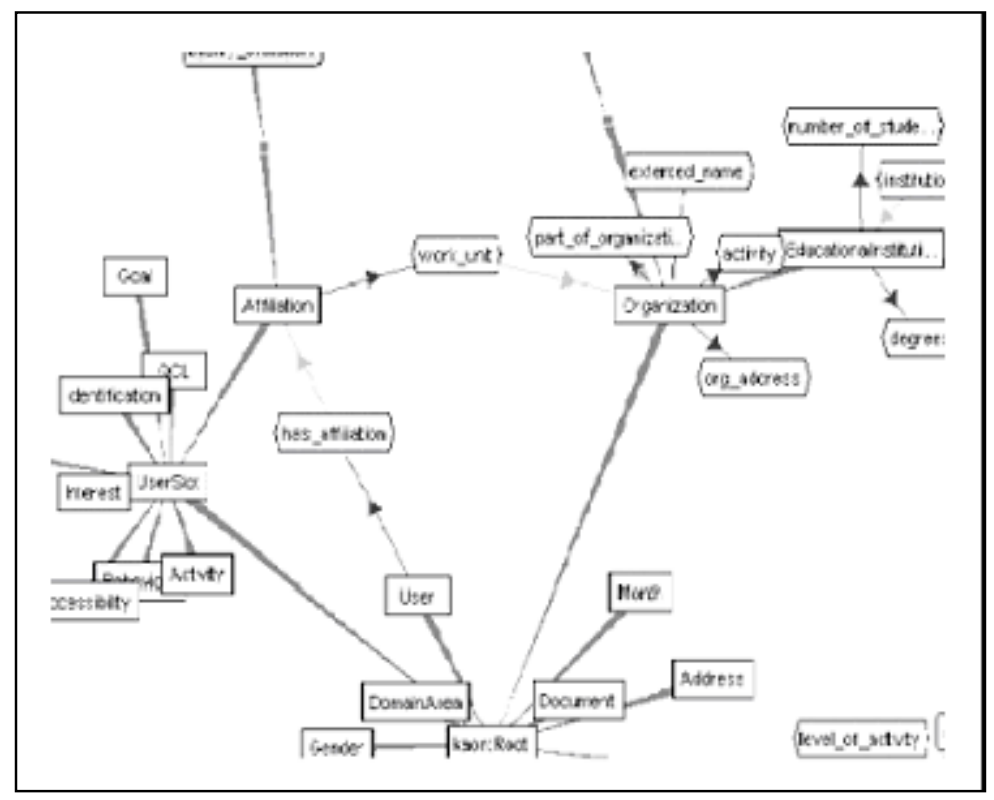

Figure 1 :A partial graph-based view on the user ontology

For the ASIMIL scenario only a part of the proposed user ontology is necessary. In a second phase the user ontology has to be mapped to the domain ontology. Ontology based user modeling requires a referential structure which can be static (e.g subconcept Name of the IMS LIP abstract concept Identifiction) and an adaptive part which in a learning context need to evolve according to the user's progress in learning, according to his goal, domains of interest which need to be acquired and updated (concepts like Interests, Goals). The dynamic part of the e-Portfolio is based on the achieved learning goal of the user and the Peer-to-Peer review of his updated abilities. For example if the learner wants to train himself to pilot an airplane (described as the learner's goal in the user ontology), he will be able to access different flight simulations amongst which also ASIMIL.(Formal versus informal learning).

\section{ASIMIL USE CASE: A NETWORK DISTRIBUTED SIMULATOR TRAINING SYSTEM}

The experimental framework for the ASIMIL Training system is Simulation-based Intelligent A Peer-to-Peer-Review process which is performed by autonomous agents (as Knowledge, Ergonomic, Psychologic). Each agent scans separately a common stream of messages coming from other actors (Human, intelligent agents, physical disposals). They performs coalitions to supply a given community of users (Instructors, Learners, Moderators,...) with diagnoses, advice and help among actors in the community. ASITS architecture (Agent Specification for Intelligent Tutoring systems) allows producing concurrent diagnostics in real-time, according to the Cognitive control of dialogue's modalities by ACT-R/PM model [12]:

- The agent perceives its environment continuously : PM,

- Reason for goal selection: $R$ and Reason for action Selectio: R'

Act: The selected actions are executed.

- When a coalition is gained the characteristics will be summed up in a vector which is broadcast to other agents and coalitions for searching P2P cooperation and bests overlays

- The final matchmaking process results from the moderation between coalitions proposals by subjective clustering.

- When an assessment is carried out by one of the actors, he/it can recover the chunk to update his knowledge and an alarm is broadcast to launch the P2P review again.

A dedicated P2P-Agents architecture for perception and qualification of erratic user's behaviours has been constructed which consists of a cognitive monitoring based on intelligent agents. (see figure 2).

${ }^{6}$ tool suite for ontology management (Maedche et al., 2002)[6] 


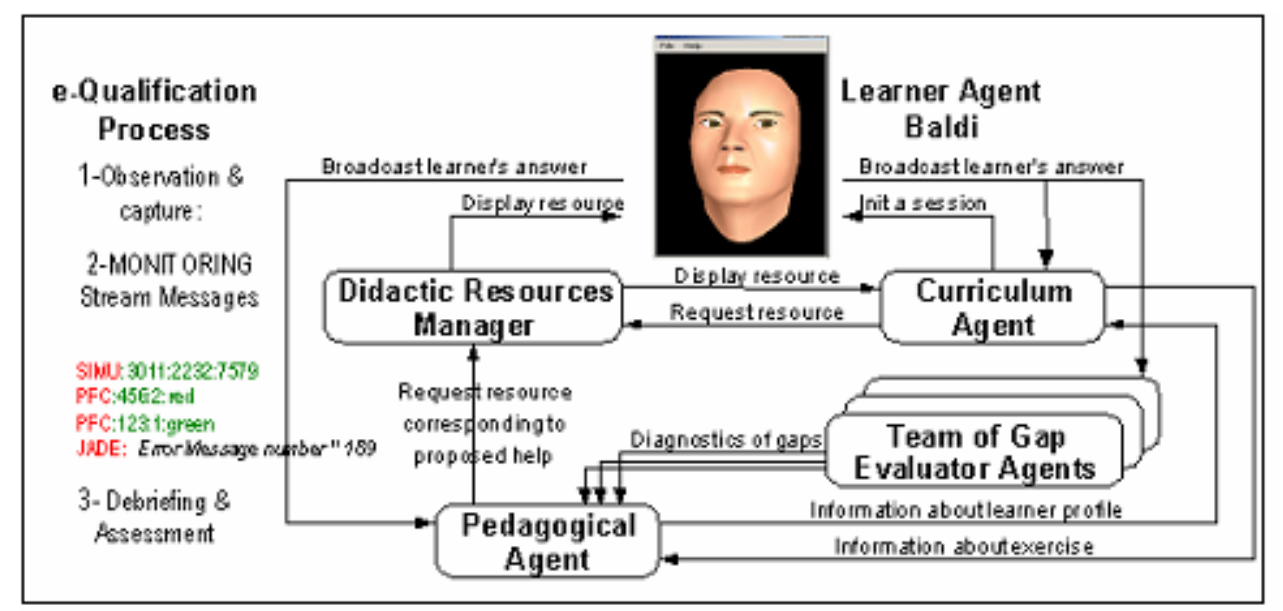

Figure 2 :e-Qualification Process in ASIMIL

\subsection{The EleGI Version}

The greatest innovation in this ASIMIL version will be the use of virtual communities that we will call "Virtual Learning among Group Communities » (VLGC). The VLGC that we use to realize the e-Qualification process is a little bit different from the VLC implemented for EleGI. Moreover, we have choose to work in a Peer-to-Peer way. So, the main characteristics of the VLGC are :

- this is an aggregation of people that want to reach the same goals ("group thinking").

- the integration or the sacking of a new trainee must be a decision made by the whole VLGC (no centralization)

- the management of the VLGC is realized with the use of e-Portfolios.

The major point is that all the members of the VLGC must have the same objectives and some common skills (necessary bases). This common point of a VLGC's members is represented by an extract of the e-Portfolio that we call the signature. The main rule is that every members of a VLGC must share a common signature.

If all this information is replace into the context of ASIMIL, the first rule is that every trainee has to belong to a community to use this simulator. Every user has a e-Portfolio. The VLGC will be managed with P2P agents. The informal part of the learning process will happen during the debriefing. The scenario is the following one : after having some instructions during the briefing, the trainee performs the exercise/simulation. At the end of this simulation, his e-Portfolio is updated according to his formal results (scoreline). That leads to a change of VLGC or not. Then debriefing occurs (in group so in VLGC) which is the informal part of the learning process. All is monitored by agents. If the end of the debriefing, the trainee has acquired (or not) some new skills. But it can be checked only by the participation to a new test. If the test is better succeed, it means that the trainee has learned in an informal way. The e-Portfolio is updated and that is what we call « the informal learning treatment ».

The use of ontologies is of course essential for this new version of ASIMIL for three major reasons :

- to allow a user to belong to several VLGC,

- to ensure the interoperability on the GRID (too many platforms, programs and programmers...),

- to allow a dynamical use of the e-Portfolio

The static part of the e-Portfolio is divided into six categories which contain several concepts as described in the following table (cf. figure 3).

\begin{tabular}{|c|c|c|}
\hline & Tables & Description \\
\hline Trainee & Trainee & information about the trainee (name, age, address, etc.) \\
\hline Strategy & Strategy & $\begin{array}{l}\text { level of the trainee and attributes which charactarize the transition from a level } \\
\text { to another one }\end{array}$ \\
\hline \multirow{2}{*}{$\begin{array}{l}\text { History (session } \\
\text { history) }\end{array}$} & Pedagogy & store the headings \\
\hline & traineeAction & store the record interactions \\
\hline \multirow{2}{*}{$\begin{array}{l}\text { Currieulum } \\
\text { (lecture }\end{array}$} & Cursus & store the heedings \\
\hline & Curitem & store the pointers toward exercises and the dependance links between exercises \\
\hline \multirow{2}{*}{\begin{tabular}{l|} 
Errors (patterns of \\
clustered erros)
\end{tabular}} & ErrorOngin & description of the errors \\
\hline & ErrorQualification & qualification of the errots \\
\hline \multirow{3}{*}{ Tolerance } & debriefV ariable & parameters and variables storage \\
\hline & Threshold & authorized levels of errors \\
\hline & PhraseType & Baldi's typical sentenses \\
\hline
\end{tabular}

Figure 3 : Concepts

The corresponding ontology is represented by figure 4. If we detail this ontology, we can easily see how it will be used by the agents. Let us take the case of the pedagogic agent or the ergonomic one that are detecting and interpreting the errors. They will use some instances of the errorOrigin or errorQualification concepts (represented figure 5). The way of using these ontologies will be described in a following paragraph (see § 4.3). 


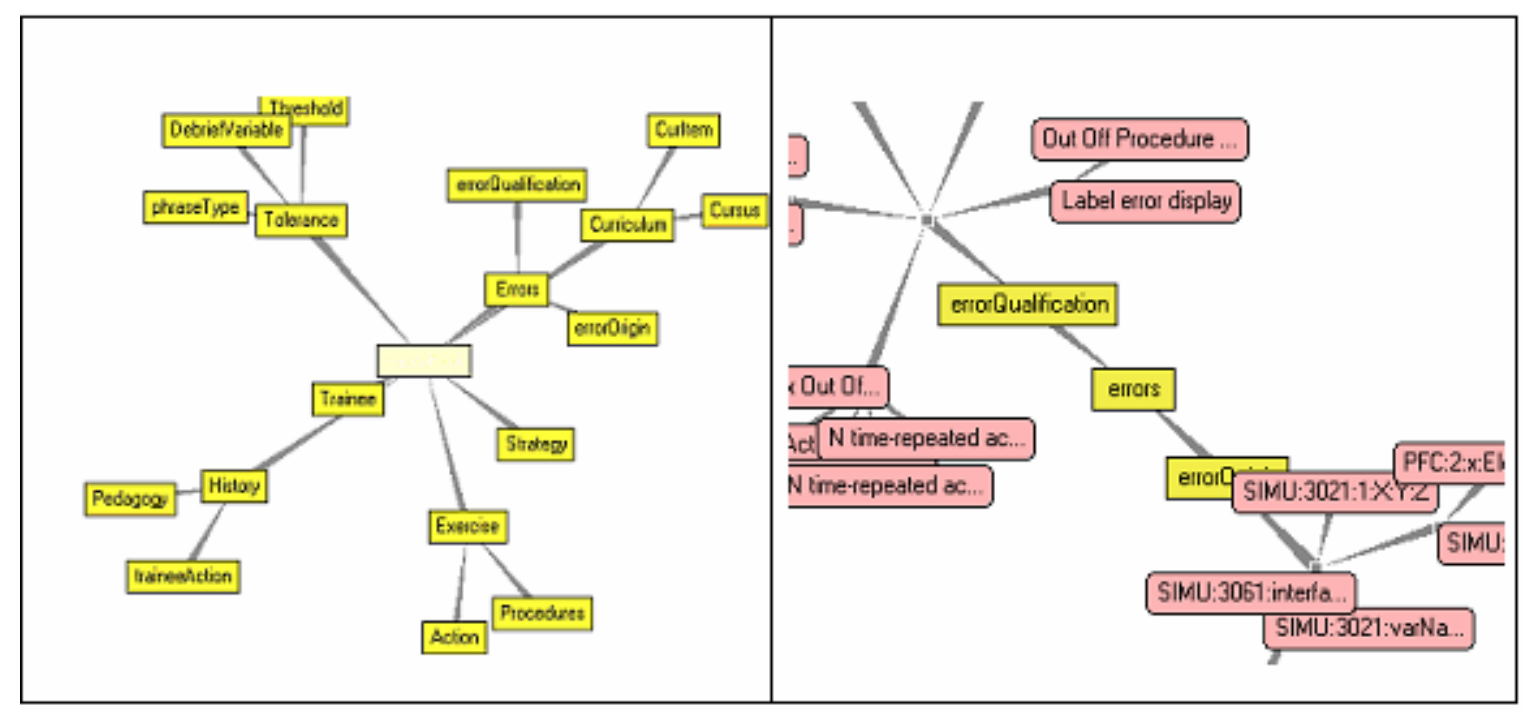

Figure 4 : e-Portfolio's Ontology

Figure 5 : Instances of the error concept

\subsection{How to use P2P agents for monitoring VLGC}

There are several needs for managing VLGCs in a decentralized manner using P2P agents. The following list describes two of them and how we try to address those needs. Note that we will use agents from Madkit[7] and JADE[8] platforms. We will use the Madkit 4.1version that provides mobility aspect for agents. We implemented required P2P methods in the two platforms using JXTA tool [9] in order to allow agents to behave in a P2P way. Using JXTA allows us making agents from different platforms to interact and communicate. In fact peerAgents will use their own platform messaging services while working on a classic way and will use JXTA implemented methods while working as Peers with other agents [10].

Dynamic and decentralized upgrade of the VLGC signature

The members of the same VLGC share a common knowledge (signature of the VLGC); this signature is modified and updated over time, as a result of the members' interactions. We need a mechanism to upgrade conditions allowing agent members (and therefore students members) to stay in the VLGC without the help of a centralized part. In fact, we want to know if all the members are aware of those changes in the signature and if they are able to understand and support the new requirements. For this task, agents will interact in a P2P manner. As soon as a pair of agent members of the VLGC upgrade their knowledge and consider that this upgrading is useful for the VLGC, all the other members will receive a message indicating that signature has changed. This has implications on some members who will not be able to satisfy the new requirements in the VLGC signature and will have to move to another VLGC that suits their profile better. However, if the member is still able to stay in the VLGC, he will modify his vision of the VLGC signature locally and continue his work inside the VLGC.

Joining the VLGC in a decentralized manner

A mechanism is required to allow students (precisely their agents) to join VLGC without invoking a centralized service. P2P technology addresses this need with the ad hoc peer administrator. One or many peer members in a PeerGroup will play the role of administrator and will be invoked when a new candidate desires to join the group. We will use the same strategy. The main condition allowing a new student to join the VLGC is still the "VLGC Signature". Considering that all the members of the VLGC share the same group signature, each one of them could answer the new candidate request and say wether he could join the VLGC. So if a member receives a "Join-VLGC-Request", he will compare the signature of the VLGC with the signature sent by the candidate and decide wether the two signatures match or not. A negotiation process will start before finally deciding to accept or not the new member. All the interactions between the candidate and the member will be in a peer manner without any external intervention. It means that any member of a VLGC could play the role of a group manager, and on a reception of a request to join the VLGC, one of the peer agents members will treat this request randomly.

\subsection{Example of P2P implementation of the dialog}

In this example there are four agents from different platforms (tow agents from Madkit platform and tow agents from JADE platform) working in a P2P behaviour in order to identify the origin of an error occurred. Each agent will propose the origin he thinks is the real cause of the problem. We will try to explain in this paragraph how agents will be able to identify the real origin without the help of centralized service or agent. The First time the agents are launched, they adopt a Peer behaviour by implementing some services required for any peer using the JXTA platform. The agents will join the VLGC of the student, which is named AssimilVLGC in this example. We notice that the first agent launched will not find the VLGC, so he will create it. We suppose that all the other agents of the ASSIMIL simulator used by the student will join this VLGC. Imagine now that the error "PFC:2X:ElementClicked" is detected, four agents will propose different possible causes for that error. Every peerAgent will broadcast his proposal to the whole VLGC. An agent who will receive the message and who hasn't proposed any possible cause will ignore this message. But for the other agents they will compare the reasons they proposed with the causes received by the other peers. If there is a matching between the two proposals, the two agents will form a couple who proposes a common reason to the problem. However if the received reason doesn't match the agent's own reason, he will ignore the message. In this example JadePeerAgent1 (JPA1) proposes "a Mouse-Click has ticked off on a wrong area from the SIMU-or-PFC " reason, he will send a broadcast message and wait for an answer. At the same time JadePeerAgent2 (JPA2) will send his own proposal which is "a Mouse-Click with the "left button " or a twice Click has ticked off a wrong item from the SIMU" and will receive a message from the JadePeerAgent1 
but will ignore it. A Few seconds later, MadkitPeerAgent3 (MPA3) and MadkitPeerAgent4 (MPA4) receive the messages from the tow former agents, and each of them will find a matching with one of the received messages. MadkitPeerAgent3 will detect that the message received from JadePeerAgent1 matches his proposition and decide to form a pair with this agent. the two agents will propose the following error reason : "Mouse-Click \& SIMU-orPFC".

The MadkitPeerAgent4 will detect that his proposal match the reason proposed by JadePeerAgent2 best.The pair will propose a common reason to the problem which is: "Mouse-Click"\& Button".(see figure 6).

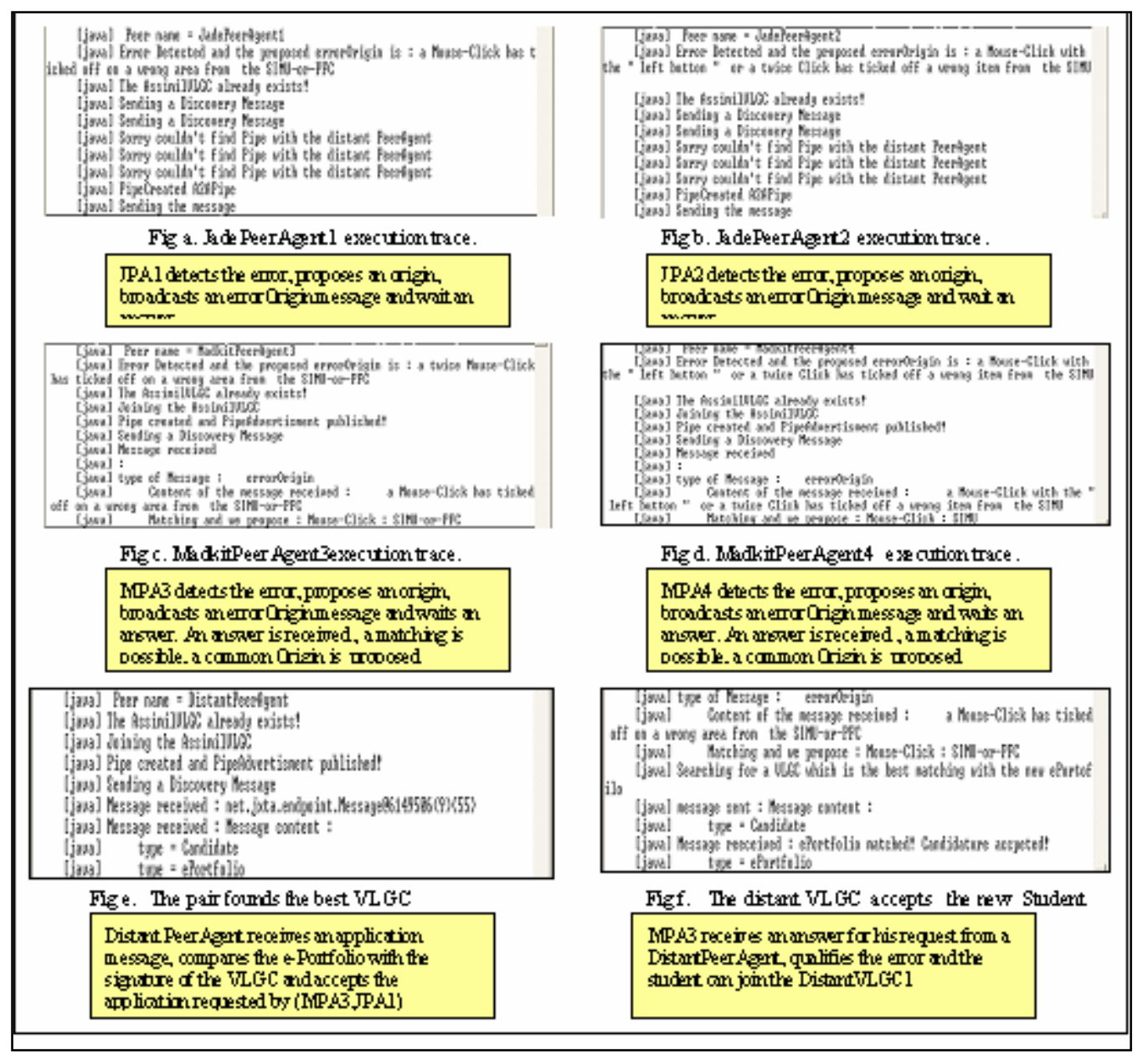

Figure 6 : Illustration of a Peer-to-Peer dialogue

The two pairs will locally modify a copy of the e-Porfolio of the user with the proposed solution for this context. With this modification they will try to found a VLGC that have the same signature as the e-Portfolio proposed by the pair. Randomly, one of the two pairs will broadcast a message to the known distant VLGCs. In the distant VLGCs one peerAgent will receive a message of application sent by the pairs. He will compare the ePorfolio sent with the signature of its VLGC and decide wether the student joins the VLGC or not. (see figure 7) 


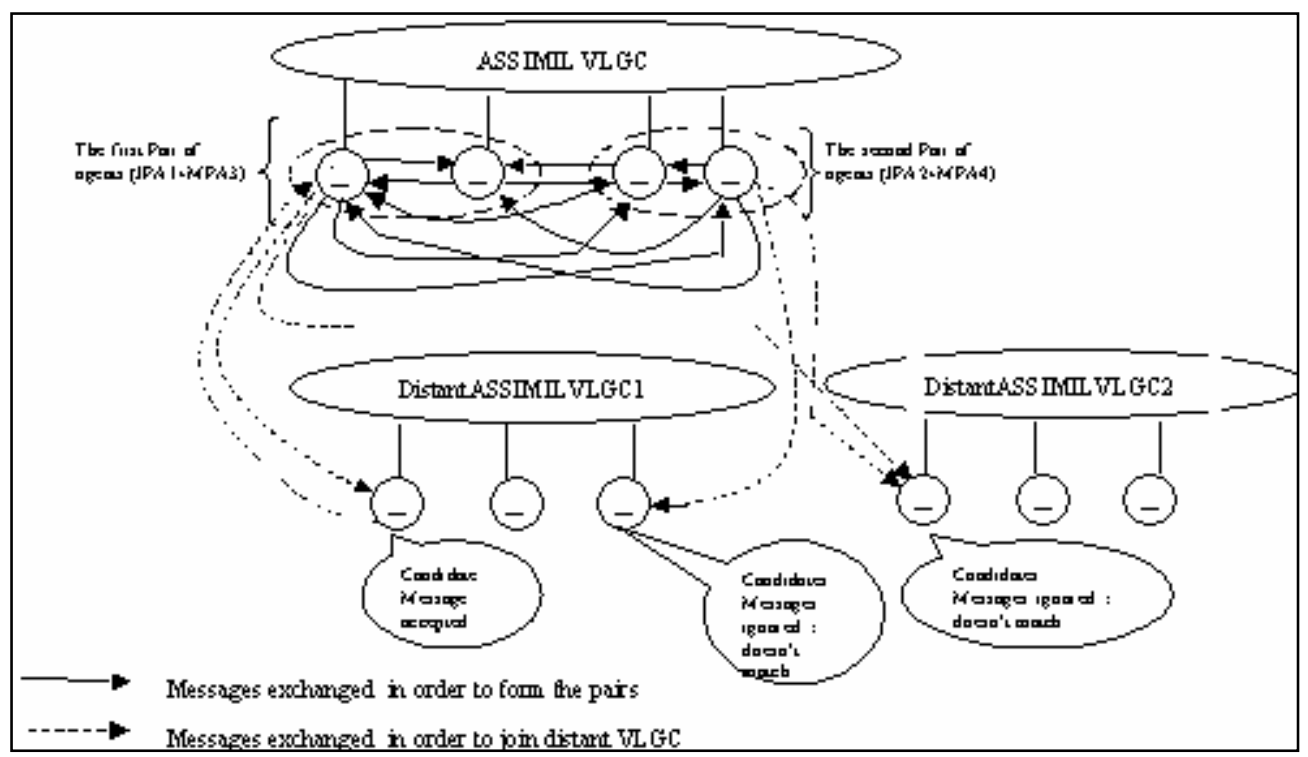

Figure 7 : e-Qualification Process with P2P agents

\section{CONCLUSION}

The paper has presented a set of technology cores that are ready to be applied for the new e-Qualification GLS. Personalization and contextualization requires access to the e-Portfolio as an associated user modeling processes or learning about the users. Ontologies are the most suitable representation mechanism integrating the semantics of dialogues between peers in VLGC. Ontology based user modeling requires a referential structure which can be static but also an adaptive part which need to evolve according to the user's progress in learning. In order to integrate these two functions and to better support their users, signatures need to be constructed and maintained by mobile agent making Peer-to-Peer review among VLGC. Personalization reduces the information overflow by providing users with the most relevant information whereas contextualization helps fitting the functionality of the system to the user's context and needs; From the e-Qualification perspective, personalization helps bridge the gap between the "designer's view of the validation of ability" and the end-user's view of his own performance and take into account the user's preferences for his well suited posting into the appropriate VLGC.

\section{ACKNOWLEDGEMENTS:}

«Work partially supported by the European Community under the Innovation Society Technologies (IST) programme of the $6^{\text {th }}$ Framework Programme for RTD - project ELeGI, contract IST-002205. This document does not represent the opinion of the European Community, and the European Community is not responsible for any use that might be made of data appearing there in. »

\section{REFERENCES}

[1] Allison, C., Cerri, S.A., Gaeta, M., Ritrovato, P. and Salerno, S.(2003), Human Learning as a Global Challenge: European Learning Grid Infrastructure. in Varis, T., Utsumi, T. and Klemm, W. eds. Global Peace Through the Global University System, RCVE, Tampere, 465-488

[2] Gouardères, G., Minko, A., Richard, L., (2000). Simulation and Multi-Agent Environment for Aircraft Maintenance Learning, actes du congrès $9^{\text {th }}$ International Conference on Artificial Intelligence: Methodology, Systems, Applications, Varna, Bulgaria, Lecture Notes in Artificial Intelligence \#1904, September 20-23, pp. 152166, Springer ed.,

[3] Yatchou, R., Tangha, C., Gouardères, G., (2004)Ubiquitous Knowledge Prosthesis for Grid Learning Services: The Grid-e-Card, International Workshop on Grid Learning Services, $7^{\text {th }}$ International Conference, ITS 2004, Maceió, Alagoas, Brazil, August 30 - September 3, Gouardères, G., Nkambou, R. (Edts), Proceedings , pp 24-38

[4] Yathiraj, B. Udupi, Munindar, P. Singh (????) Agent-Based Peer-to-Peer Service Networks: A Study of Effectiveness and Structure Evolution,: Dept of Computer Science North Carolina State U Raleigh, NC 27695, USA, Pýnar Yolum : Dept of Artificial Intelligence Vrije U, Amsterdam The Netherlands.

[5] Razmerita, L., Gouardères, G.,(2004) Ontology based user modeling for personalization of grid learning services. International Workshop on Grid Learning Services, $7^{\text {th }}$ International Conference, ITS 2004, Maceió, Alagoas, Brazil, August 30 - September 3, G. Gouardères, R. Nkambou (Edts), Proceedings , pp 105-115

[6] Razmerita, L., Angehrn A. \& Maedche, A., (2003). Ontology-based user modeling for Knowledge Management Systems, in Proceedings of "UM2003 User Modeling: Proceedings of the Ninth International Conference", Pittsburgh, USA, Springer Verlag, pp.213-217. 
[7]MadKit 4.0 (release 4.1) http://www.madkit.org/

[8] JADE (Java Agent DEvelopment Framework) version 3.2 http://jade.cselt.it/

[9] Project JXTA v2.0: Java ${ }^{\text {TM }}$ Programmer's Guide Sun Microsystems May 2003, http://www.jxta.org/

[10] Chen, Rita Yu and Yeager, Bill (2002), Java Mobile Agents on Project JXTA Peer-to-Peer Platform, ,

Sun Microsystems Proceedings of the $36^{\text {th }}$ Hawaii International Conference on System Sciences (HICSS'03)

[11] Barrett, Helen C. (2004). Differentiating Electronic Portfolios and Online Assessment Management Systems, Conference SITE , Atlanta, Georgia, March 1-6,

[12] Anderson, J. R. (1996). ACT: A simple theory of complex cognition. American Psychologist, 51, 355-365.

[13] C. Goble, D. DeRoure, ( 2004), The Semantic Grid: Myth Busting and Bridge Building," ECAI-2004, Valencia, Spain 